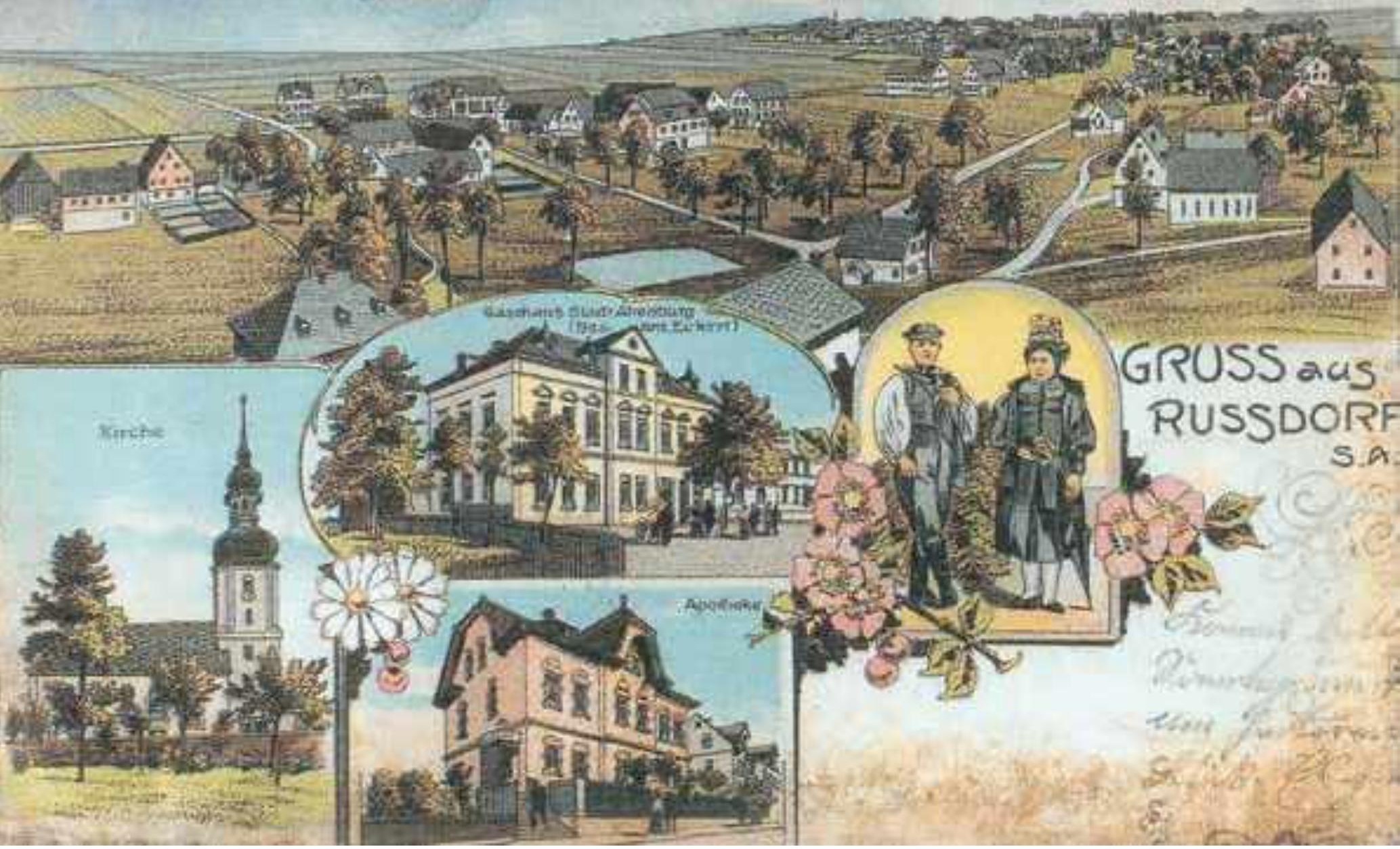

\title{
Rußdorf, ehemals eine Exklave Sachsen-Altenburgs
}

\author{
Peter Barth
}

Gruß aus Rußdorf, Postkarte, um 1900
Die älteste Geschichte des Ortes liegt im Dunkeln. Es kann davon ausgegangen werden, dass Rußdorf im Zuge der Besiedlung unserer Heimat durch rheinfränkische Siedler im 12. Jahrhundert seinen Anfang genommen hat. Die urkundliche Erwähnung im 15. Jahrhundert lautet auf Rudiger-, Rudigestorf, Rudelsdorff, bezeichnet also eine deutsche Anlage eines Rüdiger.

Rußdorf kam durch ein Tauschgeschäft zu Sachsen-Altenburg. Hildebrand von Einsiedel vertauschte das Dorf an das Kollegiatstift St. Georg in Altenburg. Die Übergabe fand am 7. Juli 1457 in der St. Georgenkirche auf dem Schloss zu Altenburg im Beisein der Stiftsherren des Kollegiatkapitels St. Georg und ihres Propstes statt. Aus der Stiftszugehörigkeit ergab sich, dass Rußdorf, trotz seiner Entfernung zu Altenburg, bei der Landesteilung zwischen Kurfürst Ernst und Herzog Albrecht am 26. August 1485 auf der ernestinischen Seite verblieb. Nach Auflösung des Kollegiatstifts infolge der Reformation kam das Dorf zum Amt Altenburg, das von 1554 bis 1918 zum Herrschaftsgebiet der ernestinischen Wettiner in Thüringen gehörte. 1603 wurde das Herzogtum Sachsen-Altenburg gegründet, dem Rußdorf bis zum Ende der Monarchie angehörte.

Das Amt Altenburg übte seit der Reformation die Ober- und Erbgerichte über das Dorf aus und es hatte sich im „hiesigen Orte ein besonderer Gerichtsstuhl erhalten“. Dazu kam das erforderliche Amtspersonal alle drei Jahre nach Rußdorf und hielt im Gasthof im Beisein der Ortsgerichtsperson Rügegericht. Verhandelt wurden Angelegenheiten von minderer Bedeutung, Sachen der freiwilligen Gerichtsbarkeit und Gemeindeangelegenheiten. Im Garten des Gasthofes befand sich auch das Gefängnis. Noch früher wurden auch alle „peinlichen“ Exekutionen im Dorf vollstreckt. Dabei hatten die Dorfbewohner die Kosten zu tragen und auch das Holz für die Säulen des Galgens zu liefern. Gerichtsschreiber bei den Verhandlungen war der jeweilige Schullehrer des Ortes. Regelmäßi- 


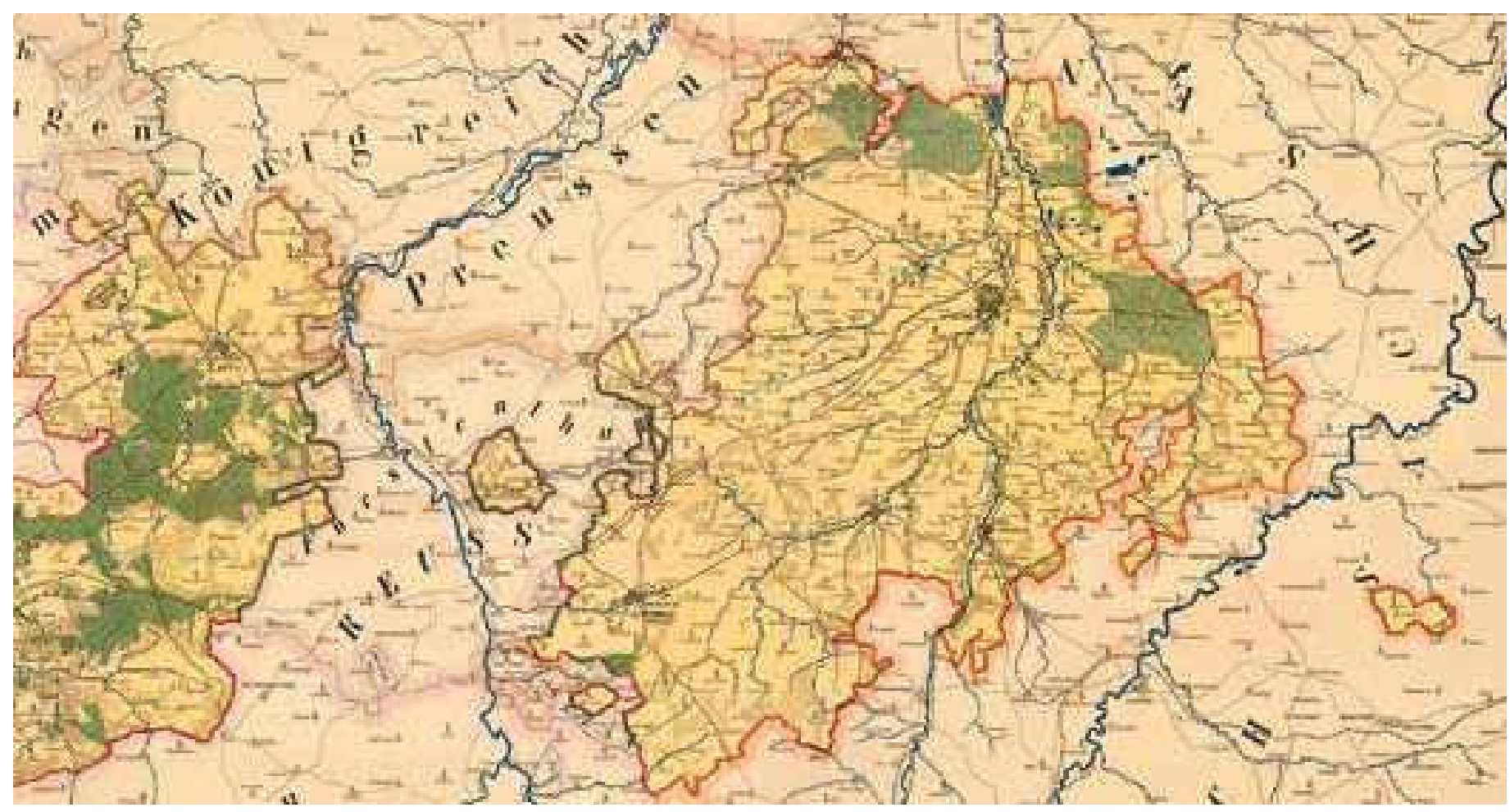

ge Gerichtstage wurden bis 1928 abgehalten.

Um die Grenze zu Sachsen festzuschreiben, wurden 1864 um die Rußdorfer Flur 314 Grenzsteine auf einer Länge von ca. 13 Kilometer gesetzt. Heute sind nur noch ein Drittel der Steine vorhanden. Viele sind im Erdreich versunken, wurden umgepflügt bzw. entfernt. Die Steine, meist aus Granit oder Glimmerschiefer, sind auf der thüringischen Seite mit einem HA für Herzogtum Altenburg und auf der sächsischen Seite mit einem KS für Königreich Sachsen sowie einer fortlaufenden Nummer gekennzeichnet.

Um das Leben der Rußdorfer durch die Grenzlage zu Sachsen nicht noch mehr zu beschweren und Kosten von den Einwohnern fernzuhalten, gab es eigene Einrichtungen. So erhielt Elisabeth Sebastian 1719 die Konzession zum Betreiben einer Wassermühle auf Rußdorfer Flur zur Grenze nach Pleißa. Die Mühle war mit einem herzoglich-altenburgischen Privileg versehen, damit die Rußdorfer Bauern ihr Getreide nicht „im Ausland“ mahlen lassen mussten und ihr Geld somit im Lande blieb. Der letzte Pächter kündigte 1875 und die Mühle wurde schließlich 1878 abgerissen. Einzige Zeugen sind heute noch die zwei Mühlteiche und die alte Linde. Die Zugehörigkeit zum Amte Altenburg führte aber auch $\mathrm{zu}$ erheblichen Behinderungen im Verkehr zwischen Rußdorf und den sächsischen Nachbarorten. Mitten im Dorf stand die Zollschranke, an der jeder Fremde eine Abgabe zu leisten hatte. In Stelzmanns Gasthof war ein Fenster, durch das ein löffelartiger Gegenstand herausgehalten wurde und in den die Zollpfennige eingelegt werden mussten. Viele benachbarte Gemeinden verlangten die Aufhebung dieser Maßnahme. Besonders heftige Streitigkeiten brachen zwischen Rußdorf und Meinsdorf aus, in deren Verlauf 1858 mehrmals der Schlagbaum gestohlen wurde.

Aber nicht nur, dass Zoll erhoben wurde, auch andere kuriose Begebenheiten ergaben sich durch die Grenzlage. So verlief die Grenze zu Sachsen entlang der heutigen Waldenburger Straße. Sowohl auf altenburgischer als auch auf sächsischer Seite gab es Gasthäuser, die unterschiedliche Sperrstunden hatten. Wollte man sein Bier länger genießen, ging man einfach nach der Sperrstunde in Sachsen über die Straße nach Altenburg und konnte eine Stunde länger im Gasthaus sitzen. Der Besitzer des Gasthofes „Jägerhaus“ erhielt 1893 die Konzession zum Ausschank von Kaffee und Altenburger
Karte des Herzogtums SachsenAltenburg, 1894, Ausschnitt des Westteils. Die Exklave rechts ist Rußdorf.

๑ Thüringisches Staatsarchiv Altenburg, Karten- und Plansammlung, Nr. 3769-3771

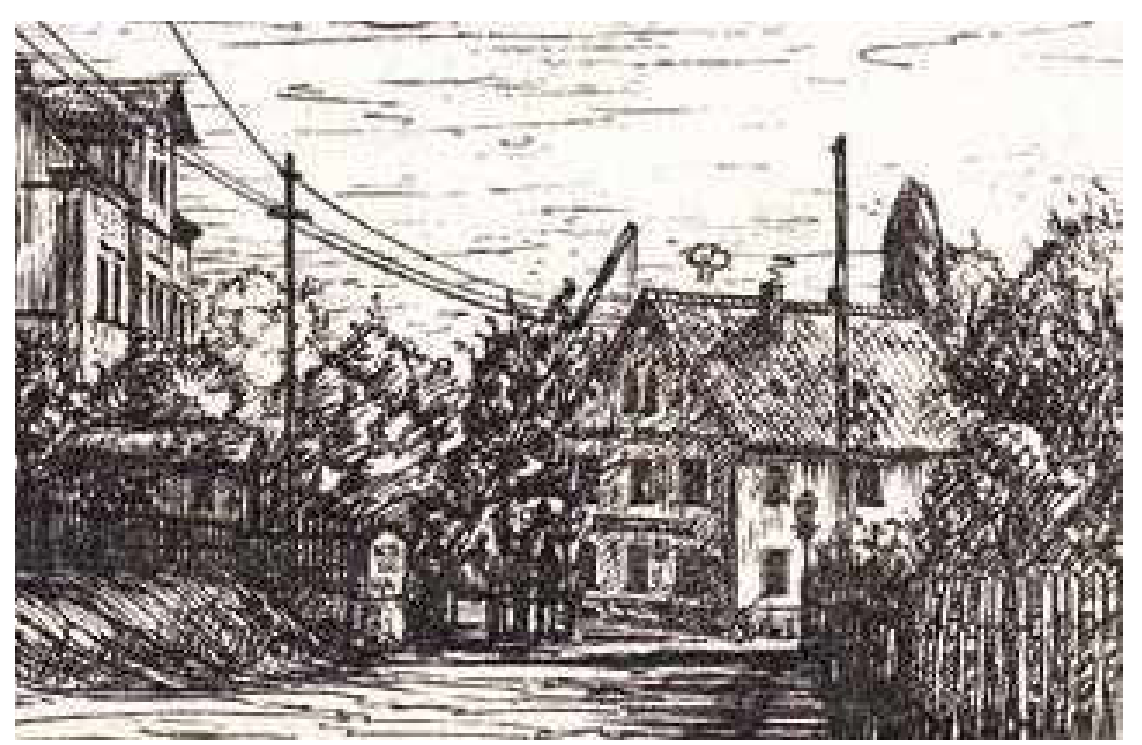




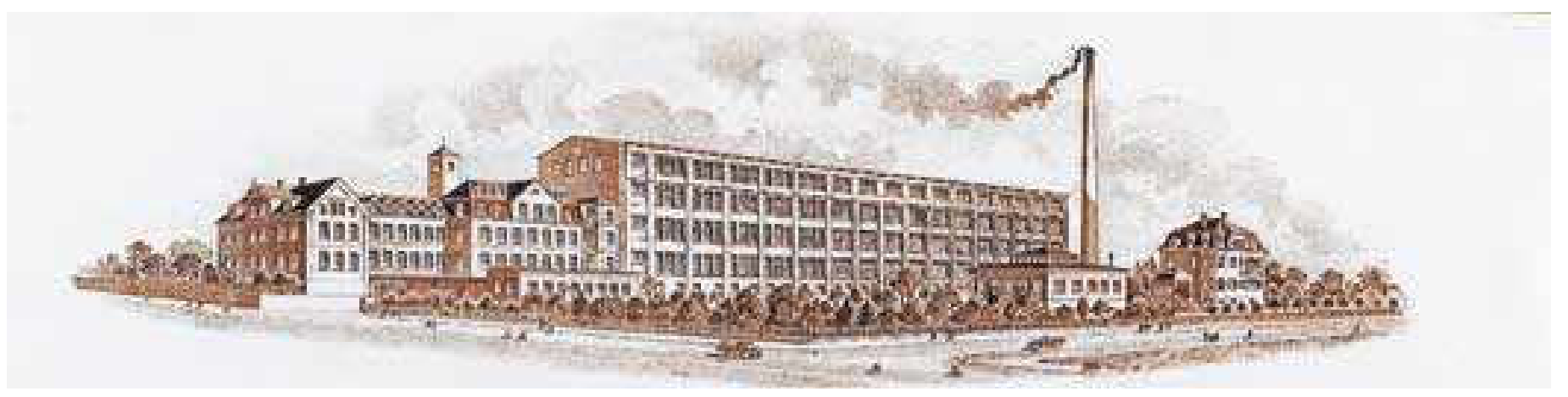

Ansicht der Wirkerei Welker \& Söhne, um 1900
Bier mit der Begründung, dass die Rußdorfer ihr Geld nicht in das benachbarte Gasthaus tragen sollten, das unmittelbar daneben auf sächsischer Seite stand.

Grenzstreitigkeiten gab es immer wieder. Ein großes Problem trat auf, als die Firma Wünschmann Anfang des 20. Jahrhunderts seine Färberei erweitern wollte, ohne die Grenze zu Limbach im Blick zu haben. Mit der Grenzüberbauung beschäftigte sich lange Zeit das Amtsgericht Altenburg. Man einigte sich schließlich auf einen Flächenaustausch.

1910 wurde dann die Zollschranke aufgehoben und der Verkehr war nunmehr ungehindert möglich. Heute erinnert auf dem Gelände des Autohauses Schmidt eine symbolische Schranke an den einstigen Standort.

Rußdorf war zunächst hauptsächlich von der Landwirtschaft geprägt. Durch zugezogene Handwerker entwickelte sich die Industrie immer weiter und die Bevölkerung wuchs in gleichem Maße rasch an. Gab es 1445 zunächst nur 10 Höfe, waren es 1535 bereits 23. Handwerker galten als freie Einwanderer. Leineweber mochte man in vielen Städten nicht und so siedelten sie sich vorwiegend auf dem Lande an. So auch Strumpfwirker und Leineweber in Rußdorf. Das ursprüngliche Bauerndorf entwickelte sich immer mehr zum Industriedorf. Mitte des 18. Jahrhunderts, hielten sich Landwirtschaft und Gewerbe fast die Waage. Um diese Zeit gab es rund 50 Bauernwirtschaften und ca. 30 kleinere, mittlere und größere Gewerbebetriebe. Die Einwohnerzahl wuchs von 706 (1791) auf 1.781 (1880) an. Der größere Teil der Einwohner nährte sich „besonders von der Fabrikation leinerner, wollener und baumwollener Waren. Am zahlreichsten sind die Strumpfwirker“.

Die Lage Rußdorfs brachte für Handwerker und alle Einwohner besondere Schwierigkeiten mit sich. So mussten die Strumpfwirker ihr Meisterrecht in Chemnitz erwerben und auch dorthin ihre Handwerksabgaben entrichten. Um dieser Erschwernis abzuhelfen, suchten drei Meister, Johann Daniel Esche, Gottfried Kretzschmar und Gottfried Müller 1745 bei Herzog Friedrich III. von Sachsen-Gotha-Altenburg um Errichtung einer eigenen Innung in Rußdorf nach, die ihnen auch gewährt und deren Artikel am
2. März 1745 bestätigt wurden. Es war die erste Strumpfwirkerinnung im Altenburgischen und auch im Limbacher Land, denn die Limbacher Innung wurde erst 40 Jahre später gegründet. Die Rußdorfer Innung bat 1755 darum, auch „an Strumpfwirker im benachbarten Dorfe Limbach das Meisterrecht ertheilen, insgleichen gegen die Pfuscher in den altenburgischen Gränzdörfern ein Verbietungsrecht üben zu dürfen. Daß die Rußdorfer sowohl in Seide als Wolle schöne tüchtige Waren fabrizierten, also daß sie mit ersterer sogar viele solche Höfe z. B. Gotha, Dresden, Weimar versähen. Sie verfertigten damals außer Strümpfen, Handschuhen und Mützen, auch Beinkleider, sonstige Kleidungsstücke und viele feine Arbeiten und der starke Warenvertrieb brachte Nahrung in den Ort, weshalb sich auch die Zahl der Meister fortwährend mehrte."

Mit Schiebböcken wurden die Waren zu den Märkten nach Glauchau, Zwickau und Altenburg transportiert und dort verkauft. Die Rußdorfer Strumpfwirkerinnung bestand bis 1901 . Sie musste aufgelöst werden, da es in Rußdorf nur noch wenige Meister gab und sich ein großer Teil der Strumpf- und Handschuhproduktion nach Oberfrohna und Limbach verlagert hatte.

Im Umfeld der Strumpf- und Handschuhfabriken siedelten sich weitere Industrien an, z.B. eine Färberei oder Metallfabriken, die Zulieferer für die nötigen Maschinen waren. 1912 bestanden in Rußdorf bereits elf Handschuhfabriken, sechs Strumpffabriken, eine Färberei und Appreturanstalt, fünf Maschinenfabriken und eine Metallfabrik. Daneben siedelten sich viele Handwerker, Händler und kleinere Geschäfte an. Es waren Namen wie Kadelbach, Alban Curt Müller, Kohlsdorf, Engelmann, Püschmann oder Geißler, Preßler \& Co., Niekamp, Sonntag oder Ihle, die noch manchem Rußdorfer in Erinnerung sind. Zu den bedeutendsten Strumpffabriken gehörte aber die 1848 gegründete Firma Welker \& Söhne, die in den 1930er Jahren bis zu 500 Arbeiter beschäftigte.

Alle diese Firmen boten den Einwohnern über viele Jahre Lohn und Brot, zum Teil auch in Heimarbeit. Es gab immer ein Auf und Ab in der Konjunktur mit zeitweise hoher Arbeitslo- 
sigkeit. Manche Firmen gingen schon vor dem Ersten Weltkrieg oder in den 1920er Jahren in Konkurs. Weitere Betriebe wurden in den 1970er Jahren verstaatlicht bzw. die Produktionsstätten von Rußdorf nach anderen Orten verlagert. Nach 1990 stellten auch die letzten Textilproduzenten in Rußdorf ihren Betrieb ein.

Mit Gaststätten war Rußdorf immer sehr gut versorgt. In den 1930er Jahren konnte man vom Ober- bis zum Unterdorf allein 15 Mal einkehren. Bereits 1582 wird ein „Gastwirt Sebastian“ erwähnt, der an der Hauptstraße ein Erbgut betrieb und zudem das Amt des Richters innehatte. 1855 wurde der "Gasthof Rußdorf“ gebaut. Auch die Gerichtstage fanden hier statt.. Mehrere Pächter betrieben ihn. Der Gasthof war Versammlungsort vieler ansässiger Vereine, es gab Tanz- und Ballmusik zu den verschiedendsten Anlässen. Auch im Gasthof „Stadt Altenburg" konnte und kann man heute noch gut einkehren.

Reges Leben brachten die vielen Vereine ins Dorf. 1913 gab es ca. 24 davon, u. a. zwei Turnvereine, Gesangsvereine, Militärvereine, auch eine Freiwillige Feuerwehr. Bis heute bereichern Vereine das Leben im Ort mit verschiedenen Aktivitäten.

Bereits sehr zeitig gab es im Ort eine Schule. Eine erste Erwähnung finden wir schon 1587 als „eine Wohnung des Küsters mit einem Grasgärtlein“. Der Küster war zugleich Lehrer und Gerichtsschreiber bei den Rügegerichten. Er wurde sowohl in Geld als auch in Naturalien entlohnt. Es war genau aufgezeichnet, wer wie viel zu liefern hatte. Für die Kinder musste je nach Bildungsstand Schulgeld gezahlt werden. Für ein Kind, das nicht schreiben konnte, waren 4 Pfennig wöchentlich und für ein Kind, welches schreiben konnte, 6 Pfennig zu entrichten. Die Zahl der Bevölkerung und somit auch die Kinderzahl nahm durch die Industriealisierung rasch zu. 1830 wurde eine neue Schule gebaut, das heutige Pfarrhaus. Auch diese Räumlichkeiten wurden zu eng, es gab inzwischen über 200 Kinder. 1868 wurde die „Alte Schule“, die nicht mehr in Betrieb ist, und schließlich 1889 die heutige „Thomas-Müntzer-Schule“, eingeweiht. Sie ist heute noch Grundschule für Rußdorf und umliegende Orte.

Erwähnenswert ist ebenfalls das heutige „Sonnenbad“. Der Gedanke eines Natur- und Heilbades entstand mit dem Anwachsen der Industrie und der Bevölkerung in der zweiten Hälfte des 19. Jahrhunderts. Es gab große Familien, die in beengten Wohnverhältnissen lebten. Auch den Arbeitern in den Strumpffabriken sollte die Möglichkeit einer gesunden Lebensweise geboten werden. Durch Aufenthalt in der Natur, mit Wasserkuren, Sonnenbädern, Bewegungstherapie und natürlichen Mitteln der Gesundheits-

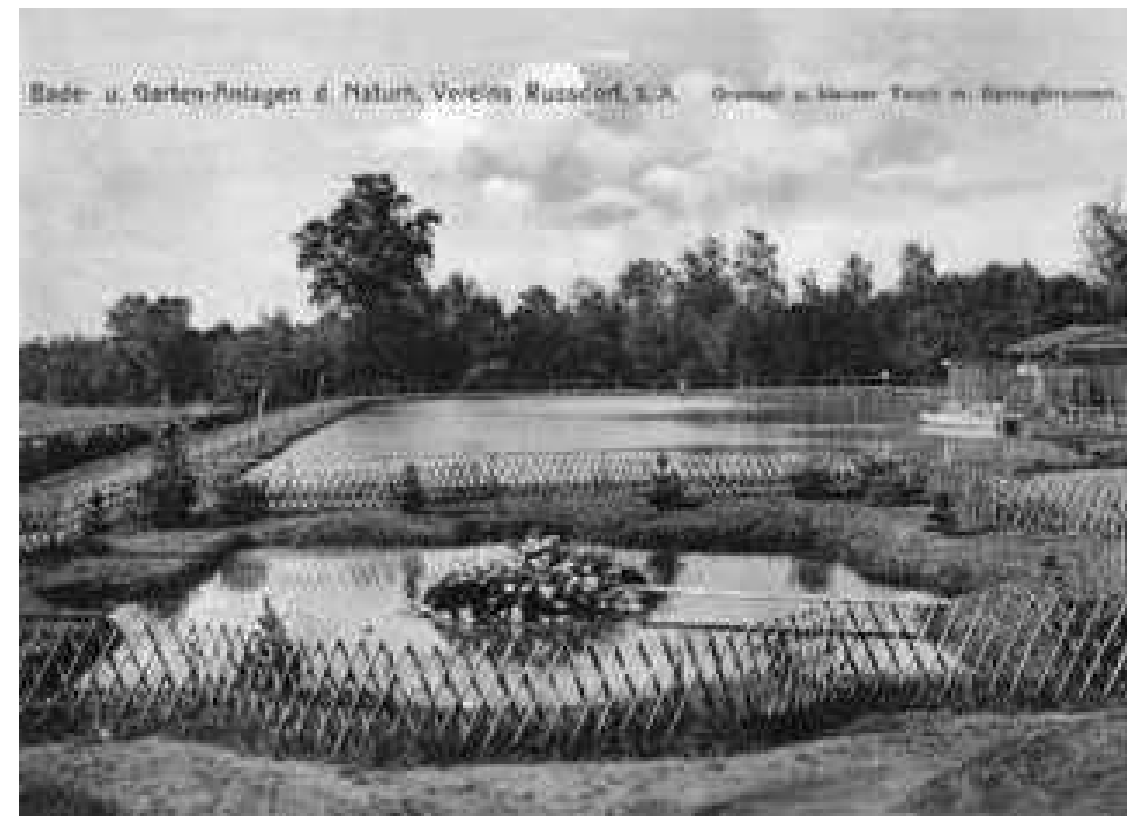

förderung sollten die Menschen bessere Bedingungen zur Erhaltung ihrer Gesundheit finden. 1889 wurde der Naturheilverein gegründet, der sich die genannten Ziele in seine Satzung geschrieben hatte. Zunächst wurden an die Mitglieder Dampfkästen, Bade- und Sitzwannen ausgeliehen. Zur Anlage eines Badeteiches mit Sonnenbad wurde ein Grundstück am Gemeindewald gekauft. Das Bad konnte 1905 eingeweiht werden. Die Mitglieder des Vereins bauten das Gelände immer weiter aus und es entwickelte sich zu einem beliebten Freizeitparadies für alle Rußdorfer. Zur weiteren Gesundheitsförderung wurden auch Parzellen zum Gemüseanbau eingerichtet. Heute finden wir an diesem Platz das „Sonnenbad“ und mehrere Kleingartenanlagen. Das Bad wird weiter ausgebaut und verschönert und ist heute Anlaufpunkt für Gäste aus Nah und Fern.

Ebenso wie der Ort hatte auch die Kirche eine wechselvolle Geschichte. In Altenburg selbst, das zum ernestinischen Sachsen gehörte, war man schon 1522 evangelisch. Bis zur Reformation war Rußdorf kirchlich mit dem benachbarten Bräunsdorf verbunden. Es gab zwar eine Filialkirche, aber der Pfarrer saß im Schönburgischen Bräunsdorf. Die Bräunsdorfer widersetzten sich der Einführung der lutherischen Lehre und Predigt, so dass sich Rußdorf 1533 von Bräunsdorf trennte und evangelisch wurde. Rußdorf wurde der kursächsischen Kirchengemeinde Kaufungen zugeschlagen, die bereits 1528 das evangelische Bekenntnis eingeführt hatte.

In der Folgezeit kam es zwischen beiden Gemeinden zu Streitigkeiten, u. a. wegen der Unterhaltung des Pfarrhauses in Kaufungen. Die Rußdorfer beteiligten sich vorerst an den Kosten unter der Bedingung, sich jederzeit von Kaufungen lösen zu können. Im Jahre 1866
Sonnenbad des Naturheilvereins Rußdorf, Postkarte, um 1900 
Johanniskirche in Rußdorf

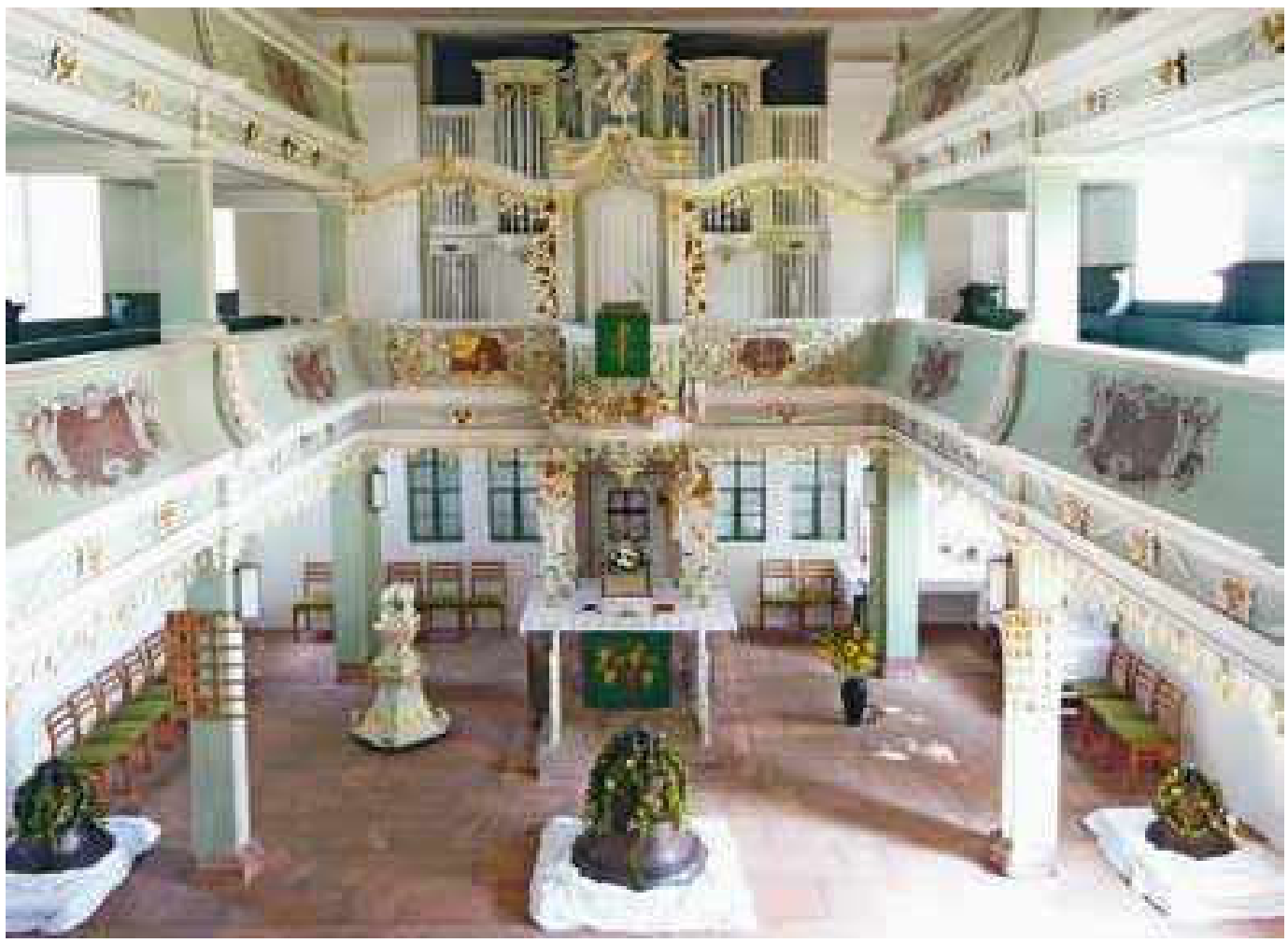

wurde dieser Beschluss verwirklicht. Nachdem auch die kirchliche Oberbehörde dazu ihre Zustimmung gegeben hatte, wurde am 4 . Sonntag nach Trinitatis 1869 der neue Pfarrer eingeführt und Rußdorf eine eigenständige Kirchengemeinde. Ein besonderes Kleinod in Rußdorf ist die barocke Johanniskirche. Sie wurde 1729 bis 1734 anstelle eines Vorgängerbau errichtet, der wegen Baufälligkeit abgerissen werden musste. Nach der Wende wurde die Kirche über viele Jahre grundlegend saniert und erstrahlt heute wieder in altem Glanz.

Das Gesicht von Rußdorf veränderte sich ständig. Es wurde neu gebaut oder abgerissen, Straßennamen geändert. Fabrikanten bauten eigene Wohnhäuser oder erweiterten ihre Betriebsstätten. 1890 war die Straße von Altrußdorf bis Limbach durch Ansiedlung von neuen Firmen oder anderen Neubauten nahezu geschlossen. 1902 wurde die Straße von Rußdorf nach Meinsdorf gebaut. Die Hohle, heute Hohe Straße, wurde immer mehr verfüllt. 1912/13 entstanden die Gemeindehäuser an der heutigen Meinsdorfer Straße Richtung Sonnenbad. In den 1930er Jahren wurden in der Wiesenstraße 30 Wohnungen geschaffen. Im Zuge des Randsiedlungsbau der Hitlerjahre entstanden an der heutigen Folgenstraße sechs Doppelhäuser. Hier konnten die Eigentümer neben einem Garten auch einen kleinen Stall bewirtschaften. Dort hielten sie vorwiegend Ziegen, deshalb nannten die Rußdorfer diese neue Siedlung auch „Ziegenschweiz“.

Als am 1. Mai 1920 der Freistaat Thüringen als politische Einheit geschaffen wurde, blieb Ruß- dorf weiterhin als Exklave Thüringens bestehen. Nach Verhandlungen zwischen den Freistaaten Thüringen und Sachsen wurde 1928 ein Staatsvertrag geschlossen, der einen beidseitigen Austausch von Exklaven vorsah. Thüringen erhielt das „Ziegenhierd'sche Ländchen“ um Liebschwitz bei Gera, während Rußdorf an Sachsen abgetreten wurde. Am 1. April 1928 erklärte der Gemeinderat in „Stelzmanns Gasthof" unter Vorsitz von Bürgermeister Schulze und in Anwesendheit von Landrat Böhme die Übergabe an Sachsen. Die Einwohner Rußdorfs waren nunmehr Sachsen. Heute erinnert nur noch der Name des Gasthofes „Stadt Altenburg“ an die frühere Zugehörigkeit des Ortes zu Sachsen-Altenburg.

Für die Rußdorfer Einwohner brachte der Gebietsaustausch beträchtliche Vorteile. Für die Angliederung an Sachsen sprachen die gewachsenen industriellen Strukturen und Bindungen an die Nachbarorte Limbach und Oberfrohna, ebenso die gewachsene Arbeiterschaft, die in den benachbarten Orten arbeitete. Vorteile ergaben sich auch in der Verwaltung, doch blieb Rußdorf nur noch sieben Jahre eine selbstständige Gemeinde. Am 1. April 1935 erfolgte die Eingemeindung nach Oberfrohna. Im Zuge weiterer Verwaltungsreformen wurde Oberfrohna am 1. Juli 1950 mit Limbach zusammengeschlossen, so dass Rußdorf ein Stadtteil von Limbach-Oberfrohna wurde. Zeitweise wurde von Limbach-Oberfrohna III gesprochen.

Heute erinnern nur noch Schilder an den ehemaligen Ortsgrenzen an die Namen der ursprünglich selbstständigen Orte. 